\title{
Neuropsychological Profile and Performance Variability in Vascular Cognitive Impairment
}

\author{
Robert P. Hart ${ }^{*}$, Al M. Best ${ }^{2}$ \\ ${ }^{1}$ Department of Psychiatry, Virginia Commonwealth University Medical Center, Richmond, USA \\ ${ }^{2}$ Department of Biostatistics and School of Dentistry, Virginia Commonwealth University, Richmond, USA \\ Email: ${ }^{*}$ rhart@mcvh-vcu.edu
}

Received 6 July 2014; revised 5 August 2014; accepted 1 September 2014

Copyright (C) 2014 by authors and Scientific Research Publishing Inc.

This work is licensed under the Creative Commons Attribution International License (CC BY). http://creativecommons.org/licenses/by/4.0/

(c) (i) Open Access

\begin{abstract}
Background: Cognitive impairment from cerebrovascular disease varies in scope. Methods: We studied healthy elderly individuals and those with mild cognitive impairment (MCI) or mild dementia attributed to vascular pathology, in order to assess the diagnostic utility of intra-individual variability across neuropsychological domains and the cognitive profile of vascular cognitive disorders. A large battery of tests was used to create z-scores for seven neuropsychological domains by principle components analysis. Results: We did not find group differences in the average intra-individual variance across neuropsychological domains. We did find group differences in the within-domain variability for Language, reflecting increased performance variability in those participants with vascular pathology. There was a lower inter-correlation of domain scores in the MCI group than in the mild vascular dementia (VD) group. The MCI group had the largest deficits in Executive Function, Fluency and Memory relative to the healthy control (HC) group. The VD group evidenced a broad range of further cognitive decline relative to the MCI group, with the largest difference on Visuoconstruction. Conclusions: While intra-individual performance variability may increase early in the course of cognitive decline from vascular pathology, this is not because of greater across-domain variability or because impairments occur in only one particular cognitive domain or small cluster of inter-related domains. Early cognitive change includes decreased executive function that cannot be attributed to slow information processing or response time.
\end{abstract}

\section{Keywords}

Cerebrovascular Disorders, Mild Cognitive Impairment, Dementia, Performance Variability, Small Vessel Disease

\footnotetext{
${ }^{*}$ Corresponding author.
} 


\section{Introduction}

Cognitive impairment of presumed cerebrovascular cause has been described as "vascular cognitive impairment" (VCI; [1]) or "vascular cognitive disorder" (VCD; [2]). The scope of the problem ranges from diminished cognition identified in community samples that is attributed to deep or periventricular white matter changes [3] [4] and considered an expression of small vessel disease (SVD; [5]), to dementia from a variety of cerebrovascular disorders. The cerebrovascular disorders most often associated with vascular dementia (VD) include atherosclerosis of large and medium sized arteries, cerebral amyloid angiopathy and SVD [6]. SVD is characterized by lacunar infarcts, microinfarcts and microbleeds, predominantly in central white matter and subcortical structures like the basal ganglia, thalamus, internal capsule, brain stem, and cerebellar white matter [6]. A large proportion of individuals with VCI do not meet criteria for dementia [7] and are diagnosed with mild cognitive impairment (MCI) using a variety of definitions of impairment (e.g., different cut-offs relative to normative data and different number of low scores overall and/or within a cognitive domain). Longitudinal studies of patients with vascular MCI demonstrate high rates of incident dementia during ensuing years, although many do not progress to dementia [8]. A recent prospective longitudinal study of a community sample indicated that increased white matter lesion (WML) burden was associated with steeper cognitive decline over the same interval [9].

Patients with VCI exhibit a broad range of cognitive impairment [10]-[15]. Among the tests most sensitive to impairment in vascular MCI as well as VD were those assessing psychomotor or processing speed (with or without set-shifting), memory, and verbal fluency. A similar pattern has been described in patients with atherosclerotic disease, with or without manifest cerebrovascular disease [16]. In the latter study, WML burden and the presence of multiple lacunar infarcts were most strongly associated with performance on a timed test of cognitive flexibility. Verbal fluency, a test assessing "the capacity to discover logical rules", and to a lesser extent verbal learning, were also associated with WML burden and/or lacunar infarcts.

Studies of patients diagnosed with probable VD and studies of patients diagnosed with either probable VD or probable Alzheimer's disease (AD; [11] [17] [18]) have shown that an increased volume of WMLs on brain MRI is associated with greater cognitive impairment. Cohen et al. [11] found the strongest association between volume of hyperintensities and performance on tests of psychomotor/processing speed (Digit Symbol) and tests of psychomotor speed that also required set-shifting (Trail-Making B) in his study of VD patients. The findings were inconsistent for measures of memory, visuospatial abilities, and verbal fluency.

The association between WMLs and cognition has been investigated in community samples of neurologically normal adults [3] [4] [19]; broadly representative community samples of mostly healthy adults [9]; stroke-free patients treated for hypertension [20]; and other samples of healthy adults [21]. While comparison of the findings across these studies is complicated by the use of different tests and different operational definitions of cognitive domains, several reliable trends are apparent. The strongest and most consistent correlates of increased WML burden were found on measures of processing and/or psychomotor speed. Some of the strongest relationships with lesion burden were found on tests that required a combination of processing speed and an aspect of executive function (e.g., Stroop interference measures) or psychomotor speed and an aspect of executive function (e.g., Trail-Making B). Executive function tests that did not require cognitive or psychomotor speed (e.g., card sorting tests, mazes) were generally not related to lesion burden. Findings were inconsistent for measures of attention/working memory, verbal fluency, and visuospatial/visuoconstructional skills. Verbal memory was not related to lesion burden, but visual memory was related to lesion burden in about half of these studies.

Recent cross-sectional and longitudinal studies suggest that intra-individual test score variability may provide important diagnostic and prognostic information for patients presenting with cognitive decline. Schretlen [22] assessed elderly individuals referred for a dementia work-up using 13 measures derived from six tests, and stratified the patients by scores on the Mini Mental State (MMSE; [23]). In addition to a decline in mean scores across groups with lower MMSE scores, the standard deviation (SD) for the demographically adjusted T-scores (reflecting intra-individual test score variability) initially increased as MMSE scores decreased, and then decreased. Specifically, relative to healthy adults, the SD was larger in the clinical sample having MMSE scores in the range of 28 - 30 and still larger in those with MMSE scores in the range of 24 - 27. The SDs then decreased as MMSEs decreased (groups with MMSE scores of 20 - 23, 16 - 19, and 9 - 15). Holtzer et al. [24] studied a community sample of elderly that excluded individuals judged to have dementia during screening. Participants were tested every 12 to 18 months on individual measures of vocabulary, psychomotor/processing speed, and verbal learning. Greater intra-individual test score variability was associated with increased risk of dementia at 
follow-up. Increased variability predicted the development of both incident AD and incident VD.

Neither of the latter two studies was intended to assess intra-individual variability across neuropsychological domains, which may also have diagnostic utility. The pattern of cognitive change across domains in MCI vs. mild dementia was not specifically identified. To date, only the study of Holtzer et al. [24] has examined intra-individual variability in patients with cerebrovascular disease, and this was limited to variability across three individual test scores in those later identified with incident VD.

The present study builds on these intriguing findings by assessing intra-individual variability across neuropsychological domains rather than individual tests in patient groups with vascular MCI and mild VD. The first aim of the study was to explore the diagnostic utility of intra-individual domain variability. The second aim was to further elucidate the pattern of change in neuropsychological functioning early during the course of vascular cognitive disorders.

\section{Methods}

\subsection{Participants}

The participants were 55 elderly individuals from a larger group of patients presenting with cognitive decline by self or informant report who were referred for neuropsychological evaluation. The individuals selected for the study group were those who evidenced cognitive impairment that was of presumed vascular etiology on the basis of clinical history, neurologic examination, and neuroimaging studies. Although cognitive decline was suspected by referring physicians, some participants $(n=8)$ were judged to have performed within the normal range on neuropsychological tests and are included to represent the range ofindividuals who presented for evaluation and for comparison to those with clinical diagnoses of MCI or mild dementia. Fifty-six percent of participants were male and 78\% were Caucasian. They were between the ages of 60 and $92(\mathrm{M}=74.6, \mathrm{SD}=6.9)$ and completed between 8 and 18 years of formal education $(M=14.9, S D=2.9)$. Medical history, laboratory studies, mental status exam, neurologic examination, and clinical interview of the patient and informant at the time of testing were used to rule out non-progressive neurologic disorders (e.g., traumatic brain injury), developmental disorders (e.g., mental retardation, learning disorders), systemic medical illness sufficient to cause cognitive decline, major psychiatric disorders, and cognitive impairment that was attributed to substance abuse. Individuals were excluded from data analyses if: 1) English was not their primary language; 2) they had a stroke causing a hemispheric syndrome that affected test-taking (e.g., aphasia, hemiparesis, hemi-spatial neglect); or 3) they were too impaired to complete the entire protocol.

Participants who evidenced cognitive impairment attributed to vascular pathology on the basis of neuroimaging, clinical history and neurologic examination were diagnosed with MCI $(n=27)$ or mild dementia $(n=20)$ on the basis of neuropsychological test performance. The clinical criteria for MCI were similar to the comprehensive criteria of Jak et al. [25] in that participants diagnosed with MCI had multiple low test scores, and in contrast to those with mild dementia, had fundamentally intact instrumental activities of daily living based on informant report and normal range scores on a test of adaptive knowledge and skills [26]. The diagnosis of MCI required more low scores (defined as greater than 1 SD below normative means) than expected on the basis of statistical probability for the number of tests administered [27]. The MMSE scores were higher in the vascular MCI group $(M=27.0, S D=1.2)$ than in the mild VD group $(M=23.7, S D=2.1)$, consistent with their respective clinical diagnoses. All healthy control (HC) subjects had MMSE scores of 28 to 30 . The assessment procedures utilized to identify cognitive decline were consistent with the standards for clinical evaluation of VCI (history-taking, neuropsychological tests, and neuroimaging studies) proposed by Hachinski et al. [28]. The neuropsychological test battery measured all of the cognitive domains identified by Gorelick et al. [29] as those that should be assessed in VCI (executive/attention, memory, language, and visuospatial functions). Individuals without a history of stroke had clinical and neuroimaging findings similar to those patients classified as non-infarct vascular dementia by Emery et al. [30]. They had a range of cardiovascular disorders and other risk factors for stroke (e.g., diabetes), as well as MRI evidence of cerebrovascular disease and ischemic features from the modified Hachinski Scale [31] such as hypertension, fluctuating disease course, and atherosclerosis, with relative preservation of personality. All of the vascular MCI and mild VD patients met the core diagnostic criteria for VCI recently proposed by Gorelick et al. [29].

This archival study using data of individuals referred for clinical evaluation was approved by the Virginia Commonwealth University Institutional Review Board. 


\subsection{Measures and Procedures}

A board-certified neuropsychologist (first author) interviewed participants and an informant (typically a spouse) and administered half of the tests. A Bachelor-level psychometrician with 25 years experience, who is a Certified Specialist in Psychometry, administered the remaining tests. The same tests were administered by the neuropsychologist and psychometrician for each participant.

Attention span and working memory capacity were assessed by Digit Span and Spatial Span from Wechsler Memory Scale-III (WMS-III; [32]). Processing speed was assessed by Wechsler Adult Intelligence Scale-III (WAIS-III; [33]) Digit Symbol and the color-word trial of the Stroop Test [34]. Memory was assessed by the incidental recall trial for Digit Symbol, WMS-III Logical Memory, Rey Auditory Verbal Learning Test [35], Continuous Visual Memory Test [36], and the 30-minute delayed recall score for the Rey-Complex Figure [37] divided by the copy score to derive a measure of percent retention. Language was assessed by selected items from the Boston Diagnostic Aphasia Exam (BDAE; [38]). Auditory comprehension was assessed by the number correct for the four paragraphs from the Complex Ideational Materials subtest (items 5 - 12). As a measure of confrontation naming, two objects (hammock, cactus), two geometric forms (star, triangle) and two body parts (elbow, wrist) from Visual Confrontation Naming subtest were presented, and each response scored 0 - 3 based on approximate response times, as indicated on the test form. Reading comprehension was assessed using all 10 items from the Reading Sentences and Paragraphs subtest. Visuoconstruction was assessed by Block Design from the Wechsler Abbreviated Scale of Intelligence (WASI; [39]) and the Rey Complex Figure. Executive functions were assessed using a modified short form of the Wisconsin Card Sorting Test [40], the errors made on the color-word trial of the Stroop Test, and a rating from 0 to 3 for each participant's copy of the Rey Complex Figure made by the first author at the time of administration. This rating was made prior to the administration of all other tests except for one measure of word recognition. Normal planning and organization was scored 0 , subtle to mild impairment was scored 1 , moderate impairment was scored 2, and severe impairment resulting in gross distortion of the figure was scored 3 . We have previously shown that lower ratings are associated with impairment in daily living skills [41]. Fluency was assessed using Animal Naming from the BDAE, FAS [42] and a measure of ideational fluency, the Alternate Uses Test [43]. The latter test was developed to assess divergent thinking. A modified version used in the present study has been shown to discriminate dementia patients from healthy elderly controls more reliably than measures of verbal fluency [44].

\subsection{Analyses}

Variable Computation. SAS software (SAS version 9.3, JMP version 11, SAS Institute Inc., Cary, NC) was used in all analyses. Raw scores from all tests were used to create z-scores in seven cognitive domains (see Table 1). Scores in each domain were created by calculating the first principal factor (principal components analysis with squared-multiple correlations on the diagonals) from all of the raw scores included in the domain. The result was a z-score representing level of impairment in each cognitive domain (negative scores indicating impairment in all domains except Executive Function). To characterize each participant's overall performance the z-scores across the seven cognitive domains were averaged, and to characterize each participant's variability in performance across domains the standard deviation of the seven z-scores was calculated.

Statistical Analyses. There are three analyses of the seven domain scores. First, the groups were compared on all demographic characteristics using a MANOVA. Then separate analyses were performed on each of the seven domains and their average in order to identify differences in group means (using Welch ANOVA) and differences in within-group variability for each domain and across domains (by the Brown-Forsythe test).Comparison of groups on variability across domains addressed the first aim of the study. In order to address the second aim of the study, a repeated-measure mixed-model ANOVA was used to compare the group mean scores across the seven domains and also to test for differences between the groups on variances and covariances. All tests were done at a 5\% significance level.

\section{Results}

The means and standard deviations of the z-scores for each cognitive domain, for each of the three groups, are shown in Table 2. A MANOVA indicated that there was no effect of age $(p=0.0885)$, sex $(p=0.1168)$, or race $(p=0.1894)$ on domain scores, but an effect of years of education $(p=0.0043)$ and group $(p<0.0001)$. That is, group differences in domain scores persisted even after accounting for differences in education. 
Table 1. Cognitive domains used to assess intra-individual variability.

\begin{tabular}{|c|}
\hline Cognitive Domain \\
\hline Attention \\
\hline Digit Span Forward/Spatial Span Forward \\
\hline Digit Span Backward/Spatial Span Backward \\
\hline Processing Speed \\
\hline Digit Symbol (sec) \\
\hline Stroop Color-Word Trial (sec) \\
\hline Memory \\
\hline Digit Symbol Free Recall/Pair Recall \\
\hline Immediate Logical Memory Recall/RAVLT Total Recall Trials 1 - 5 \\
\hline Delayed Logical Memory Recall/Delayed RAVLT Recall \\
\hline Continuous Visual Memory Test Total Correct \\
\hline Rey Figure Delay Recall $\div$ Copy Score (\% Retention) \\
\hline Language \\
\hline BDAE Complex Ideational Material (0 - 8) \\
\hline BDAE Visual Confrontation Naming $(0$ - 18) \\
\hline BDAE Reading Sentences and Paragraphs $(0-10)$ \\
\hline Visuoconstruction \\
\hline Block Design \\
\hline Rey Figure Copy Score \\
\hline Executive Function \\
\hline Wisconsin Card Sorting Test Errors/Perseverative Errors \\
\hline Stroop Color-Word Trial (errors) \\
\hline Rey Figure Rating \\
\hline Fluency \\
\hline Animal Naming/FAS \\
\hline Alternate Uses Test \\
\hline
\end{tabular}

RAVLT = Rey Auditory Verbal Learning Test; BDAE = Boston Diagnostic Aphasia Exam.

The bottom portion of Table 2 shows the "Across Domain" mean z-score for each group, and the SD reflecting intra-individual across-domain variability for each group. As expected, the groups mean scores were significantly different $(p<0.0001)$. These differences remained significant after covarying out differences due to education. The SD of across-domain z-scores did not reliably differentiate the three groups in the analysis that allowed for differences in overall mean scores $(p=0.112)$. A pre-planned contrast in across-domain variability between just the MCI and VD groups was also non-significant $(p=0.0815)$.

As shown in Table 2, for every cognitive domain there was a significant difference between the three group's means. Tukey's HSD multiple comparison procedure indicated that for every domain the three groups were statistically different from one another. Table 2 also shows the test for the hypothesis that the SDs for the three groups, representing within domain variance, are the same. There was no group difference in within domain variability for Attention, Processing Speed, Memory, Visuoconstruction, Executive Function, and Fluency. There was a difference in variability for Language scores $(p<0.001)$. Compared to the variance of the HC group, patients with vascular MCI have 15 times the variance in scores on tests of Language and patients with VD have 51 times the variance in scores on tests of Language. 
Table 2. Summary statistics for domain z-scores for HC $(n=8)$, MCI $(n=26)$, and $\operatorname{VD}(n=21)$.

\begin{tabular}{|c|c|c|}
\hline Group & Mean & SD \\
\hline & Attention & \\
\hline $\mathrm{HC}$ & 1.358 & 0.683 \\
\hline MCI & 0.208 & 0.744 \\
\hline VD & -0.774 & 0.650 \\
\hline \multirow[t]{2}{*}{$\mathrm{p}$ value } & $<0.0001$ & 0.781 \\
\hline & Processing speed & \\
\hline $\mathrm{HC}$ & 1.248 & 0.676 \\
\hline MCI & 0.230 & 0.775 \\
\hline VD & -0.748 & 0.714 \\
\hline \multirow[t]{2}{*}{$\mathrm{p}$ value } & $<0.0001$ & 0.875 \\
\hline & Memory & \\
\hline $\mathrm{HC}$ & 1.410 & 0.781 \\
\hline MCI & 0.109 & 0.663 \\
\hline VD & -0.672 & 0.808 \\
\hline \multirow[t]{2}{*}{$\mathrm{p}$ value } & $<0.0001$ & 0.737 \\
\hline & Language & \\
\hline $\mathrm{HC}$ & 0.749 & 0.171 \\
\hline MCI & 0.235 & 0.666 \\
\hline VD & -0.577 & 1.220 \\
\hline \multirow[t]{2}{*}{$\mathrm{p}$ value } & $<0.0001$ & $<0.001$ \\
\hline & Visuoconstruction & \\
\hline $\mathrm{HC}$ & 1.204 & 0.629 \\
\hline MCI & 0.363 & 0.725 \\
\hline VD & -0.909 & 0.534 \\
\hline \multirow[t]{2}{*}{$\mathrm{p}$ value } & $<0.0001$ & 0.160 \\
\hline & Executive function & \\
\hline $\mathrm{HC}$ & -1.510 & 0.353 \\
\hline MCI & -0.114 & 0.719 \\
\hline VD & 0.716 & 0.737 \\
\hline \multirow[t]{2}{*}{$\mathrm{p}$ value } & $<0.0001$ & 0.344 \\
\hline & Fluency & \\
\hline $\mathrm{HC}$ & 1.396 & 0.952 \\
\hline MCI & 0.071 & 0.626 \\
\hline VD & -0.619 & 0.840 \\
\hline \multirow[t]{2}{*}{$\mathrm{p}$ value } & $<0.001$ & 0.368 \\
\hline & Across Domains & \\
\hline $\mathrm{HC}$ & 1.268 & 0.660 \\
\hline MCI & 0.190 & 0.699 \\
\hline VD & -0.716 & 0.801 \\
\hline $\mathrm{p}$ value & $<0.0001$ & 0.112 \\
\hline
\end{tabular}

Note: Welch ANOVA was used to compare the means and the Brown-Forsythe test was used to compare the standard deviations within each group. 
The interrelationships between the cognitive domains for the entire sample are shown in Table 3 . Note that Executive Function is negatively related to the other domains because a low score represents better performance. However, the apparent correlation between cognitive domains is at least partially due to mean differences between the groups. Figure 1 shows a heat-map of the standard deviations and the correlations between domain scores for each group. If there were a common set of correlations and standard deviations, then the panel depicting the HC group would be similar to the panel depicting the MCI patients and the VD patients. A mixed-model repeated-measure ANOVA tested this hypothesis, and the likelihood-ratio chi-square comparing models with

Table 3. Pearson product moment correlations between cognitive domains, all subjects.

\begin{tabular}{|c|c|c|c|c|c|c|c|}
\hline Cognitive domain & Attention & Processing Speed & Memory & Language & Visuoconstruction & Executive Function & Fluency \\
\hline Attention & 1.00 & 0.70 & 0.63 & 0.42 & 0.66 & -0.54 & 0.56 \\
\hline Processing Speed & 0.70 & 1.00 & 0.65 & 0.52 & 0.60 & -0.58 & 0.77 \\
\hline Memory & 0.63 & 0.65 & 1.00 & 0.51 & 0.43 & -0.47 & 0.67 \\
\hline Language & 0.42 & 0.52 & 0.51 & 1.00 & 0.46 & -0.34 & 0.51 \\
\hline Visuoconstruction & 0.66 & 0.60 & 0.43 & 0.46 & 1.00 & -0.58 & 0.51 \\
\hline Executive Function & -0.54 & -0.58 & -0.47 & -0.34 & -0.58 & 1.00 & -0.65 \\
\hline Fluency & 0.56 & 0.77 & 0.67 & 0.51 & 0.51 & -0.65 & 1.00 \\
\hline
\end{tabular}

Note: All correlations statistically significant $(p<0.01)$.



Figure 1. Color map of pearson product moment correlations between cognitive domain z-scores (off diagonal) and within group standard deviation (on diagonal), separately by group.

and without a common covariance matrix indicated that the groups have a different pattern of interrelationships between the domain scores (chi-square $=124, \mathrm{df}=56, p<0.0001$ ). What is noteworthy is that for MCI patients, the correlations between domain scores are closer to zero (mean $r=0.17$ ) than for the other two groups, suggesting that within the MCI group, impairment in one domain is less related to impairment in another domain. In contrast to prior studies, Executive Function and Processing Speed were separate domains and none of the test scores for Executive Function were based on response speed. It is interesting to note that there was a group difference in the pattern of relationship between Executive Function and Processing Speed. Recall that for Executive Function a negative z-score indicates higher functioning, so this domain should be negatively correlated with all other domain z-scores. This is indicated most clearly by the blue square in the HC group, representing a negative correlation between Executive Function and Processing Speed $(r=-0.72)$. This is similar to the VD group, where the correlation is also negative $(r=-45)$, but contrasts with the small positive correlation $(r=+0.12)$ for the MCI group. 


\section{Discussion}

Healthy elderly subjects performed better on neuropsychological tests than MCI patients, who in turn performed better than mild VD patients. This is to be expected because test performance was used to make the clinical diagnoses of MCI and mild dementia. It is noteworthy nevertheless that the three groups were different from one another across all seven neuropsychological domains. This finding is consistent with prior studies demonstrating a relatively broad range of cognitive impairment in patients with VCI [10]-[16]. In these earlier studies, impairments on tests of attention/working memory and naming/verbal fluency were not consistently observed in those patients with vascular MCI [10] [12]-[14]. Relative to the HC group, we found decrements across the domains of Attention, Fluency and Language, although the change in language abilities was relatively small.

One of the two aims of the present study was to assess the diagnostic utility of intra-individual variability across neuropsychological domains in patients with milder degrees of cognitive impairment presumed to be due to vascular pathology. We did not find differences in the average variance across neuropsychological domains between the HC, vascular MCI, and mild VD groups. This does not mean that patients with incipient or mild VD do not exhibit increased variability in test performance. Holtzer et al. [24] have already demonstrated that increased within-person across-test variability is associated with an increased risk of incident VD at follow-up. The latter investigators administered individual tests of vocabulary, digit-symbol substitution, and free recall on a word list learning test. However, these three tests were not of equal difficulty level. For example, the level of performance on the vocabulary test was not associated with future dementia, which might be expected as this is a "hold" test, which is less sensitive to age-related diseases than measures of psychomotor speed and verbal learning. Thus, differences in test sensitivity could account for increased within-person across-test variability in patients with incipient VD. Our findings suggest that within-person across-domain variability has less diagnostic utility than across-test variability.

The only neuropsychological domain in which we found group differences in within-domain variability was Language. Specifically, compared to the variance in Language scores in the HC group, patients with MCI had 15 times the variance in scores and patients with mild VD had 51 times the variance in scores. Language abilities, aside from verbal fluency, as well as verbal intelligence have not been found to be reliably associated with increased WML burden [4] [9] [20]. Garrett et al. [12] found an impairment in confrontation naming in patients with VD but not in those with vascular MCI. An impairment in confrontation naming and/or comprehension in our patient groups would likely be infrequent relative to impairments in other domains more sensitive to WML burden. Sporadic language-related deficits, perhaps due to a lacunar infarct, or to microinfarcts or microbleeds, would result in greater within-domain variability. This instance of increased within-domain variability seems consistent with the across-test variability identified by Holtzer et al. [24].

In our MCI patients, the low correlations between domain scores indicates that at the earliest sign of cognitive decline from vascular pathology, impairment in one domain is less related to impairment in another domain than in those patients who have progressed to dementia. Thus, in MCI from presumed vascular pathology, there does not appear to be a select cluster of affected cognitive domains. Interestingly, MCI patients differed from the HC group and those with VD by a lack of correlation between Executive Function and Processing Speed. This may be partly due to the fact that none of the tests in our Executive Function domain were timed measures.

The second aim of this study was to further elucidate the pattern of cognitive change early in the course of VCI. Few studies have contrasted patients with clinical diagnoses of vascular MCI and VD, and those doing so used a relatively narrow range of tests compared to those employed in our study [10] [12]. Canning et al. [10] only used individual tests of semantic and phonemic fluency. They found a decrement on both tests in vascular MCI and a further decline in VD. Garrett et al. [12] found decrements in vascular MCI patients on a test of psychomotor/processing speed that also required set shifting (Trail-Making Test) as well as on a test of verbal learning, but not on tests of confrontation naming or verbal fluency. Patients with VD had larger decrements on these tests compared to those with MCI, as well as additional deficits in confrontation naming and verbal fluency.

As shown in Table 2, relative to the HC group, those with MCI had the largest deficits in Executive Function, Fluency, and Memory (1.3 to 1.4 SD). The next largest deficits were in Attention and Processing Speed (1.0 to $1.2 \mathrm{SD})$. There was the least change in Visuoconstruction (0.84 SD) and Language (0.51 SD). This broad range of cognitive change in vascular MCI is generally consistent with findings in a prior study of vascular MCI patients that also utilized a large battery of tests [13]. The relationships among the domains of Executive Function, Fluency, and Processing Speed in our MCI group are noteworthy. There was a lack of correlation between Ex- 
ecutive Function and Processing Speed in the MCI group. Prior studies of patients with VCI have included within the domain of executive function tests of verbal fluency and/or tests that also require psychomotor or processing speed such as the Trail-Making Test or Stroop Test. In contrast, the domain of Executive Function in our study did not include any timed tests. Thus, we found evidence that executive functions not requiring rapid responding are among the earliest changes from vascular pathology. Our domains that measured speed of response (Fluency, Processing Speed) were not only among the most sensitive to early change in VCI, but were the two domains most highly correlated in the MCI group (see Figure 1). Recent studies of heterogeneous samples of MCI patients using cluster and discriminant function analyses [45] [46] have identified amnestic and dysexecutive subtypes, as well as a variously defined "mixed" subgroup. In these studies, executive function tests were primarily composed of timed measures (e.g., Trail-Making B, Stroop-type tests with or without a switching condition, fluency tests with or without a switching condition). Interestingly, in the study by Delano-Wood et al. [46] the "Executive/Processing Speed" subgroup had greater WML burden than the other subgroups, which would be consistent with the pattern of our findings in the vascular MCI group.

Relative to the MCI group, those in the mild VD group evidenced a broad range of further cognitive decline (see Table 2). There was a greater degree of intercorrelation among domain scores in the VD group than in the MCI group (see Figure 1). The largest difference between the MCI and VD groups was in Visuoconstruction (1.3 SD), followed by further change in Processing Speed and Attention (approximately 1.0 SD). The other domains also showed further decline (approximately 0.7 to $0.8 \mathrm{SD}$ ). In the studies identifying MCI subgroups using cluster analysis, deficits in visuospatial abilities were most strongly associated with the dysexecutive subgroup [45] [46] which had greater WML burden [46]. Our findings suggest that impairments on tests like Block Design and the Rey Complex Figure occur relatively early in VCI, perhaps most often during a transition from vascular MCI to mild VD. Language abilities, aside from verbal fluency, appear to remain relatively more stable.

Any study attributing cognitive impairment to cerebrovascular disease is complicated by the well-established co-morbidity of VD and AD pathology, and by the often modest sensitivity of different clinical criteria for VD to pathology findings [6]. Also, while WMLs are related to SVD, non-ischemic pathological processes can be present in the same region, including degenerative changes [47] [48]. Some investigators have proposed that vascular changes may precede or accelerate AD pathology [49]. The complexity of differential diagnosis is illustrated in studies of patients diagnosed with MCI. While amnestic MCI is often a prodrome to dementia of the $\mathrm{AD}$ type, the distinction between amnestic and non-amnestic MCI subtypes does not consistently distinguish AD and vascular pathology at autopsy [50]. We found a decline in memory of greater than one SD in our group with a clinical diagnosis of vascular MCI. In a recent study of MCI patients with a high rate of family history of AD, Stricker et al. [51] found decreased WM microstructural integrity on whole-head high resolution T1 and diffusion tensor neuroimaging independent of $\mathrm{AD}$-signature cortical thinning. WM integrity was related to cognition, independent of cortical thinning, including parietal WM integrity and executive functioning as assessed by the Stroop Test, Trail-Making B, and the WCST.

Several limitations of our study should be noted. The number of healthy elderly subjects was small, although sufficient to reliably differentiate them from individuals with MCI. The vascular etiology of cognitive impairment was presumed on the basis of neuroimaging, clinical history, and neurologic examination, but the literature suggests the relationship between such clinical criteria and pathology findings is far from perfect. While neuroimaging studies indicated cerebrovascular disease, we had no quantitative measure of lesion burden. This was a cross-sectional study and thus we were not able to confirm that the MCI identified by neuropsychological tests is in all cases a prodrome to VD or any other form of dementia.

We did not examine intra-individual variability across tests within a domain, as the number of tests within a domain was as low as two. Intra-individual variability in processing speed, operationalized as trial to trial reaction time inconsistency, appears to be a sensitive marker for WM integrity and late life cognitive decline [52]. Future studies might investigate intra-individual variability across tests within the same domain, at least for processing speed.

Our study does help to clarify the diagnostic utility of intra-individual performance variability and further elucidate the pattern of cognitive change early in the course of VCI. We found that while intra-individual across-test variability may increase early in the course of cognitive decline from vascular pathology, this likely reflects differences in test sensitivity rather than early impairment in only one cognitive domain or in a small cluster of inter-related domains. While we found a broad range of impairment in vascular MCI, the deficits in 
executive function, fluency, and memory were larger than those in visuoconstruction or language. We found further broad decline in the mild VD group, including a significant decline in visuoconstruction. Finally, our study is the first to demonstrate early changes in executive function from presumed vascular pathology that cannot be attributed to decreased processing speed or slowed responding.

\section{Conflicts of Interest}

Neither author has potential conflicts of interest.

\section{References}

[1] Bowler, J.V. and Hachinski, V.C. (2003) Vascular Cognitive Impairment. Oxford University Press, Oxford and New York.

[2] Román, G.C., Sachdev, P., Royall, D.R., Bullock, R.A., Orgogozo, J.M., López-Pousa, S., et al. (2004) Vascular Cognitive Disorder: A New Diagnostic Category Updating Vascular Cognitive Impairment and Vascular Dementia. Journal of the Neurological Sciences, 226, 81-87. http://dx.doi.org/10.1016/j.jns.2004.09.016

[3] Soriano-Raya, J.J., Miralbell, J., Lopez-Cancio, E., Bargalló, N., Arenillas, J.F., Barrios, M., et al. (2012) Deep versus Periventricular White Matter Lesions and Cognitive Function in a Community Sample of Middle-Aged Participants. Journal of the International Neuropsychological Society, 18, 874-885. http://dx.doi.org/10.1017/S1355617712000677

[4] Ylikoski, R., Ylikoski, A., Erkinjuntti, T., Sulkava, R., Raininko, R. and Tilvis, R. (1993) White Matter Changes in Healthy Elderly Persons Correlate with Attention and Speed of Mental Processing. Archives of Neurology, 50, 818-824. http://dx.doi.org/10.1001/archneur.1993.00540080029009

[5] Pantoni, L., Poggesi, A. and Inzitari, D. (2007) The Relation between White-Matter Lesions and Cognition. Current Opinion in Neurology, 20, 390-397. http://dx.doi.org/10.1097/WCO.0b013e328172d661

[6] Jellinger, K.A. (2013) Pathology and Pathogenesis of Vascular Cognitive Impairment-A Critical Update. Frontiers in Aging Neuroscience, 5, 1-19. http://dx.doi.org/10.3389/fnagi.2013.00017

[7] Elias, M.F., Sullivan, L.M., D’Agostino, R.B., Elias, P.K., Beiser, A., Au, R., et al. (2004) Framingham Stroke Risk Profile and Lowered Cognitive Performance. Stroke, 35, 404-409.

http://dx.doi.org/10.1161/01.STR.0000103141.82869.77

[8] Ingles, J.L., Wentzel, C., Fisk, J.D. and Rockwood, K. (2002) Neuropsychological Predictors of Incident Dementia in Patients with Vascular Cognitive Impairment, without Dementia. Stroke, 33, 1999-2002. http://dx.doi.org/10.1161/01.STR.0000024433.36590.1B

[9] Vannorsdall, T.D., Waldstein, S.R., Kraut, M., Pearlson, G.D. and Schretlen, D.J. (2009) White Matter Abnormalities and Cognition in a Community Sample. Archives of Clinical Neuropsychology, 24, 209-217. http://dx.doi.org/10.1093/arclin/acp037

[10] Canning, S.J., Leach, L., Stuss, D., Ngo, D.L. and Black, S.E. (2004) Diagnostic Utility of Abbreviated Fluency Measures in Alzheimer Disease and Vascular Dementia. Neurology, 62, 556-562. http://dx.doi.org/10.1212/WNL.62.4.556

[11] Cohen, R.A., Paul, R.H., Ott, B.R., Moser, D.J., Zawacki, T.M., Stone, W., et al. (2002) The Relationship of Subcortical MRI Hyperintensities and Brain Volume to Cognitive Function in Vascular Dementia. Journal of the International Neuropsychological Society, 8, 743-752. http://dx.doi.org/10.1017/S1355617702860027

[12] Garrett, K.D., Browndyke, J.N., Whelihan, W., Paul, R.H., DiCarlo, M., Moser, J.D., et al. (2004) The Neuropsychological Profile of Vascular Cognitive Impairment-No Dementia: Comparisons to Patients at Risk for Cerebrovascular Disease and Vascular Dementia. Archives of Clinical Neuropsychology, 19, 745-757. http://dx.doi.org/10.1016/j.acn.2003.09.008

[13] Nyenhuis, D.L., Gorelick, P.B., Geenen, E.J., Smith, C.A., Gencheva, E., Freels, S., et al. (2004) The Pattern of Neuropsychological Deficits in Vascular Cognitive Impairment-No Dementia (Vascular CIND). The Clinical Neuropsychologist, 18, 41-49. http://dx.doi.org/10.1080/13854040490507145

[14] O’Sullivan, M., Morris, R.G. and Markus, H.S. (2005) Brief Cognitive Assessment for Patients with Cerebral Small Vessel Disease. Journal of Neurology, Neurosurgery \& Psychiatry, 76, 1140-1145. http://dx.doi.org/10.1136/jnnp.2004.045963

[15] Seo, S.W., Lee, B.H., Kim, E.J., Chin, J., Cho, Y.S., Yoon, U., et al. (2007) Clinical Significance of Microbleeds in Subcortical Vascular Dementia. Stroke, 38, 1949-1951. http://dx.doi.org/10.1161/STROKEAHA.106.477315

[16] Geerlings, M.I., Appelman, A.P., Vincken, K.L., Mali, W.P. and van der Graff, Y. (2009) Association of White Matter Lesions and Lacunar Infarcts with Executive Functioning: The SMART-MR Study. American Journal of Epidemiology, 170, 1147-1155. http://dx.doi.org/10.1093/aje/kwp256 
[17] Price, C.C., Jefferson, A.L., Merino, J.G., Heilman, K.M. and Libon, D.J. (2005) Subcortical Vascular Dementia: Integrating Neuropsychological and Neuroradiologic Data. Neurology, 65, 376-382. http://dx.doi.org/10.1212/01.WNL.0000168877.06011.15

[18] Price, C.C., Garrett, K.D., Jefferson, A.L., Cosentino, S., Tanner, J.J., Penney, D.L., et al. (2009) Leukoaraiosis Severity and List-Learning in Dementia. The Clinical Neuropsychologist, 23, 944-961. http://dx.doi.org/10.1080/13854040802681664

[19] De Groot, J.C., De Leeuw, F.E., Oudkerk, M., van Gijn, J., Hofman, A., Jolles, J. and Breteler, M.M.B. (2000) Cerebral White Matter Lesions and Cognitive Function: The Rotterdam Scan Study. Annals of Neurology, 47, 145-151. http://dx.doi.org/10.1002/1531-8249(200002)47:2<145::AID-ANA3>3.0.CO;2-P

[20] van Swieten, J.C., Geyskes, G.G., Derix, M.M., Peeck, B.M., Ramos, L.M., van Latum, J.C. and van Gijn, J. (1991) Hypertension in the Elderly Is Associated with White Matter Lesions and Cognitive Decline. Annals of Neurology, 30, 825-830. http://dx.doi.org/10.1002/ana.410300612

[21] DeCarli, C., Murphy, D.G., Tranh, M., Grady, C.L., Haxby, J.V., Gillette, J.A., et al. (1995) The Effect of White Matter Hyperintensity Volume on Brain Structure, Cognitive Performance, and Cerebral Metabolism of Glucose in 51 Healthy Adults. Neurology, 45, 2077-2084. http://dx.doi.org/10.1212/WNL.45.11.2077

[22] Schretlen, D.J. (2013) Novel Methods of Inference in Clinical Neuropsychology. Meeting of the American Academy of Clinical Neuropsychology, Chicago, IL, 19-22 June 2013.

[23] Folstein, M.F., Folstein, S.E. and McHugh, P.R. (1975) Mini-Mental State. A Practical Method for Grading the Cognitive State of Patients for the Clinician. Journal of Psychiatric Research, 12, 189-198. http://dx.doi.org/10.1016/0022-3956(75)90026-6

[24] Holtzer, R., Verghese, J., Wang, C., Hall, C.B. and Lipton, R.B. (2008) Within-Person Across-Neuropsychological Test Variability and Incident Dementia. The Journal of the American Medical Association, 300, 823-830. http://dx.doi.org/10.1001/jama.300.7.823

[25] Jak, A.J., Bondi, M.W., Delano-Wood, L., Wierenga, C., Corey-Bloom, J., Salmon, D.P. and Delis, D.C. (2009) Quantification of Five Neuropsychological Approaches to Defining Mild Cognitive Impairment. American Journal of Geriatric Psychiatry, 17, 368-375. http://dx.doi.org/10.1097/JGP.0b013e31819431d5

[26] Linkenhoker, D. and McCarron, L. (1980) Manual for Adaptive Behavior: The Street Survival Skills Questionnaire. McCarron-Dial Systems, Dallas, TX.

[27] Ingraham, L.J. and Aiken, C.B. (1996) An Empirical Approach to Determining Criteria for Abnormality in Test Batteries with Multiple Measures. Neuropsychology, 10, 120-124. http://dx.doi.org/10.1037/0894-4105.10.1.120

[28] Hachinski, V., Iadecola, C., Petersen, R.C., Breteler, M.M., Nyenhuis, D.L., Black, S.E., et al. (2006) National Institute of Neurological Disorders and Stroke-Canadian Stroke Network Vascular Cognitive Impairment Harmonization Standards. Stroke, 37, 2220-2241. http://dx.doi.org/10.1161/01.STR.0000237236.88823.47

[29] Gorelick, P.B., Scuteri, A., Black, S.E., Decarli, C., Greenberg, S.M., Iadecola, C., et al. (2011) Vascular Contributions to Cognitive Impairment and Dementia: A Statement for Healthcare Professionals from the American Heart Association/American Stroke Association. Stroke, 42, 2672-2713. http://dx.doi.org/10.1161/STR.0b013e3182299496

[30] Emery, V.O.B., Gillie, E.X. and Smith, J.A. (1996) Reclassification of the Vascular Dementias: Comparisons of Infarct and Noninfarct Vascular Dementias. International Psychogeriatrics, 8, 33-61. http://dx.doi.org/10.1017/S1041610296002475

[31] Rosen, W.G., Terry, R.D., Fuld, P.A., Katzman, R. and Peck, A. (1980) Pathological Verification of Ischemic Score in Differentiation of Dementias. Annals of Neurology, 7, 486-488. http://dx.doi.org/10.1002/ana.410070516

[32] Wechsler, D. (1997) Wechsler Memory Scale. 3rd Edition, The Psychological Corporation, San Antonio.

[33] Wechsler, D. (1997) Wechsler Adult Intelligence Scale. 3rd Edition, The Psychological Corporation, San Antonio.

[34] Golden, C.J. (1978) Stroop Color and Word Test: Manual for Clinical and Experimental Uses. Stoetling, Chicago.

[35] Rey, A. (1964) L'examen Clinique en Psychologie. Presses Universitaires de France, Paris.

[36] Trahan, D.E. and Larrabee, G.J. (1988) Continuous Visual Memory Test. Psychological Assessment Resources, Odessa, FL.

[37] Osterrieth, P.A. (1944) Le test de copie d’une figure complexe. Archives de Psychologie, 30, 286-356.

[38] Goodglass, H. and Kaplan, E. (1972) The Assessment of Aphasia and Related Disorders. Henry Klimpton, London.

[39] Wechsler, D. (1999) Wechsler Abbreviated Scale of Intelligence. The Psychological Corporation, San Antonio.

[40] Hart, R.P., Kwentus, J.A., Wade, J.B. and Taylor, J.R. (1988) Modified Wisconsin Card Sorting Test in Elderly Normal, Depressed and Demented Patients. The Clinical Neuropsychologist, 2, 49-56.

http://dx.doi.org/10.1080/13854048808520085 
[41] Hart, R.P. and Bean, M.K. (2010) Executive Function, Intellectual Decline and Daily Living Skills. Aging, Neuropsychology and Cognition, 18, 64-85. http://dx.doi.org/10.1080/13825585.2010.510637

[42] Benton, A.L. (1968) Differential Behavioral Effects in Frontal Lobe Disease. Neuropsychologia, 6, 53-60. http://dx.doi.org/10.1016/0028-3932(68)90038-9

[43] Guilford, J.P., Christensen, P.R., Merrifield, P.R. and Wilson, R.C. (1978) Alternate Uses: Manual of Instructions and Interpretation. Sheridan Psychological Services, Orange, CA.

[44] Hart, R.P. and Wade, J.B. (2006) Divergent Thinking in Alzheimer's and Frontotemporal Dementia. Aging, Neuropsychology, and Cognition, 13, 281-290. http://dx.doi.org/10.1080/13825580490904246

[45] Clark, L.R., Delano-Wood, L., Libon, D.J., McDonald, C.R., Nation, D.A., et al. (2013) Are Empirically-Derived Subtypes of Mild Cognitive Impairment Consistent with Conventional Subtypes? Journal of the International Neuropsychological Society, 19, 635-645. http://dx.doi.org/10.1017/S1355617713000313

[46] Delano-Wood, L., Bondi, M.W., Sacco, J., Abeles, N., Jak, A.J., Libon, D.J. and Bozoki, A. (2009) Heterogeneity in Mild Cognitive Impairment: Differences in Neuropsychological Profile and Associated White Matter Lesion Pathology. Journal of the International Neuropsychological Society, 15, 906-914. http://dx.doi.org/10.1017/S1355617709990257

[47] Leys, D., Pruvo, J.P., Parent, M., Vermersch, P., Soetaert, M., Steinling, M., Delacourte, A., Défossez, A., Rapoport, A. and Clarisse, J. (1991) Could Wallerian Degeneration Contribute to "Leuko-Araiosis" in Subjects Free of Any Vascular Disorder? Journal of Neurology, Neurosurgery, and Psychiatry, 54, 46-50. http://dx.doi.org/10.1136/jnnp.54.1.46

[48] Fazekas, F., Schmidt, R. and Scheltens, P. (1998) Pathophysiologic Mechanisms in the Development of Age-Related White Matter Changes of the Brain. Dementia and Geriatric Cognitive Disorders, 9, 2-5. http://dx.doi.org/10.1159/000051182

[49] Leszek, J., Sochocka, M. and Gasiorowski, K. (2012) Vascular Factors and Epigenetic Modifications in the Pathogenesis of Alzheimer's Disease. Journal of the Neurological Sciences, 323, 25-32. http://dx.doi.org/10.1016/j.jns.2012.09.010

[50] Schneider, J.A., Arvanitakis, Z., Leurgans, S.E. and Bennett, D.A. (2009) The Neuropathology of Probable Alzheimer Disease and Mild Cognitive Impairment. Annals of Neurology, 66, 200-208. http://dx.doi.org/10.1002/ana.21706

[51] Stricker, N.H., Salat, D.H., Foley, J.M., Zink, T.A., Kellison, I.L., McFarland, C.P., et al. (2013) Decreased White Matter Integrity in Neuropsychologically Defined Mild Cognitive Impairment Is Independent of Cortical Thinning. Journal of the International Neuropsychological Society, 19, 925-937. http://dx.doi.org/10.1017/S1355617713000660

[52] Nilsson, J., Thomas, A.J., O’Brien, J.T. and Gallagher, P. (2014) White Matter and Cognitive Decline in Aging: A Focus on Processing Speed and Variability. Journal of the International Neuropsychological Society, 20, 262-267. http://dx.doi.org/10.1017/S1355617713001458 
Scientific Research Publishing (SCIRP) is one of the largest Open Access journal publishers. It is currently publishing more than 200 open access, online, peer-reviewed journals covering a wide range of academic disciplines. SCIRP serves the worldwide academic communities and contributes to the progress and application of science with its publication.

Other selected journals from SCIRP are listed as below. Submit your manuscript to us via either submit@scirp.org or Online Submission Portal.
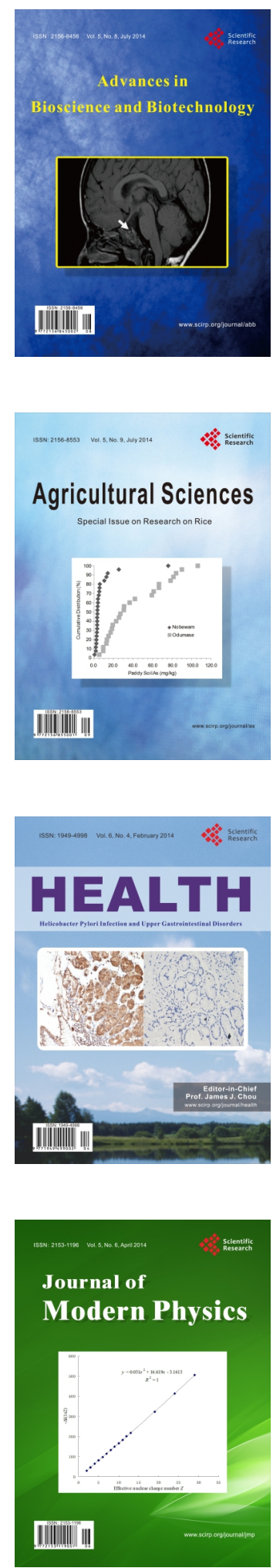
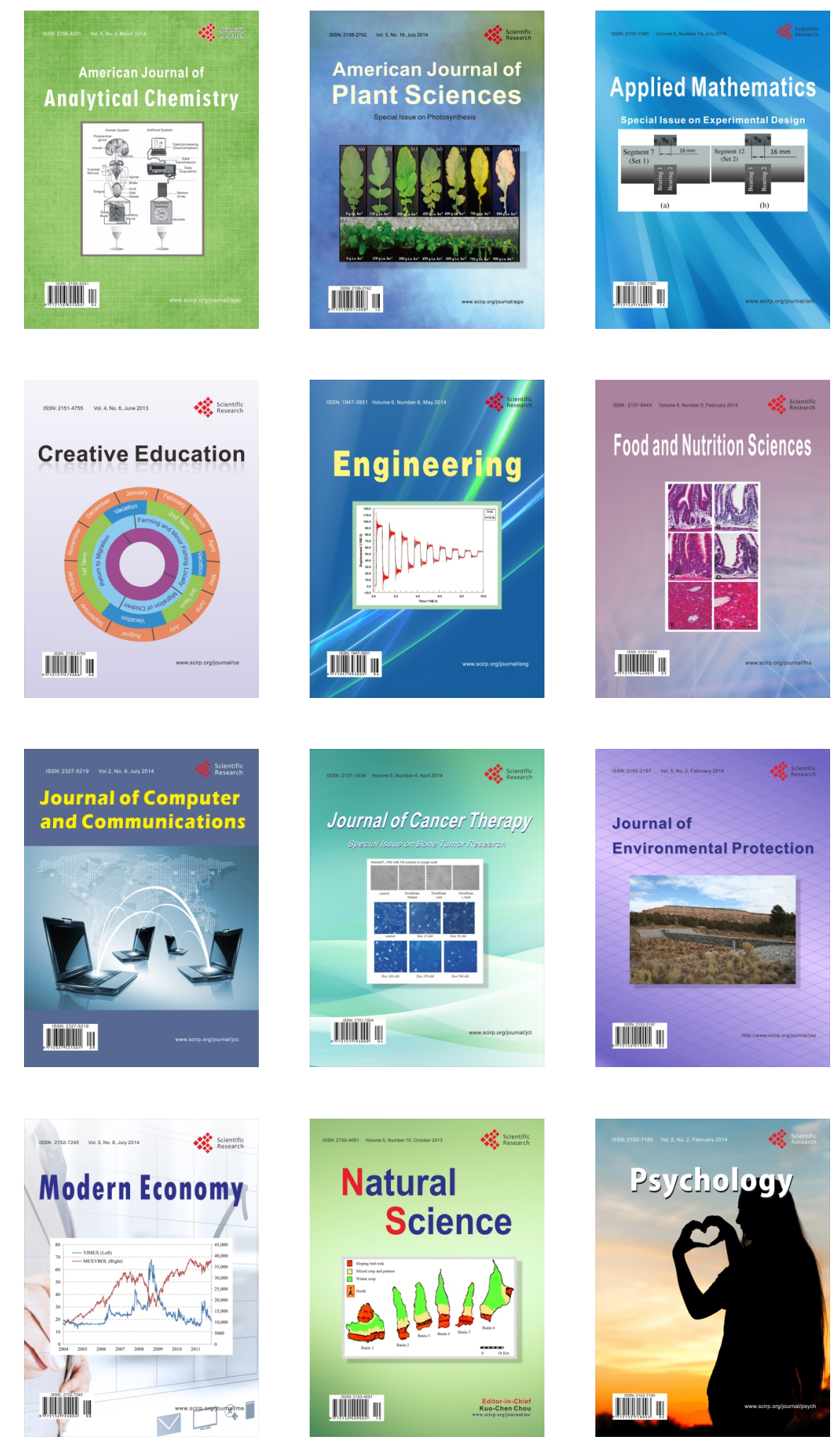